\title{
Enabling Multiphoton and Second Harmonic Generation Imaging in Paraffin-Embedded and Histologically Stained Sections
}

\author{
Michael G. Monaghan, PhD, Sebastian Kroll, BSc, Sara Y. Brucker, MD, \\ and Katja Schenke-Layland, PhD, MSc ${ }^{1-3}$
}

Nonlinear microscopy, namely multiphoton imaging and second harmonic generation (SHG), is an established noninvasive technique useful for the imaging of extracellular matrix (ECM). Typically, measurements are performed in vivo on freshly excised tissues or biopsies. In this article, we describe the effect of rehydrating paraffinembedded sections on multiphoton and SHG emission signals and the acquisition of nonlinear images from hematoxylin and eosin (H\&E)-stained sections before and after a destaining protocol. Our results reveal that bringing tissue sections to a physiological state yields a significant improvement in nonlinear signals, particularly in SHG. Additionally, the destaining of sections previously processed with H\&E staining significantly improves their SHG emission signals during imaging, thereby allowing sufficient analysis of collagen in these sections. These results are important for researchers and pathologists to obtain additional information from paraffin-embedded tissues and archived samples to perform retrospective analysis of the ECM or gain additional information from rare samples.

\section{Introduction}

$\mathbf{M}$ ULTIPHOTON-INDUCED MICROSCOPY has become an important tool in various applications, such as in vivo imaging with subcellular resolution on skin, ${ }^{1}$ early cancer diagnosis, ${ }^{2}$ evaluation of tissue-engineered products, ${ }^{3}$ and in situ drug screening, ${ }^{4}$ and for imaging extracellular matrix (ECM) structures. ${ }^{5}$ Two-photon excitation is based on the simultaneous absorption of two photons with generation of fluorescence in the visible range. This concept of quantum optics was predicted by Göpert-Mayer in her doctoral dissertation in $1931^{6}$ and first observed using laser excitation in $1961 .^{7}$ Near-infrared (NIR) light generates photons with a high concentration in space and time, which are capable of exciting fluorophores to a higher electronic state. When this photon state becomes relaxed, this leads to emission of light in a wavelength greater than one half of the excitation wavelength. ${ }^{2}$ Other microscopy techniques, such as confocal microscopy, use wavelengths in the ultraviolet (UV) spectral range. However, UV light can alter tissue structures by bleaching, polymerizing, or thermal damaging. ${ }^{8}$ Such effects do not occur with multiphoton excitation due to the low energy input of the NIR laser light.
Multiphoton excitation can enable autofluorescence of a variety of endogenous fluorophores, such as nicotinamide adenine dinucleotide phosphate (NADH) in the mitochondrial matrix, elastin in the ECM, ${ }^{5}$ or keratin in epithelial cells. ${ }^{9}$ Two ECM structures, elastic and collagen fibers, can be therefore easily imaged without the use of histological staining techniques and processing.

Mature elastic fibers are composed of elastin, which can be visualized using multiphoton imaging. Elastin is a highly hydrophobic protein formed by cross-linking tropoelastin molecules into an organized microfibrillar network, and elastic fibers are typically present in tissues that undergo stretch, such as blood vessels, lungs, and skin. ${ }^{10}$ Elastic fibers are often present in combination with collagen fibers to prevent overexpansion by restoring collagen fibers back into their original position ${ }^{11}$. Such collagen molecules consist of three collagen polypeptide chains, called $\alpha$-chains, which wind around one another in a rope-like arrangement to build a long, stiff, triple-stranded helical structure ${ }^{12}$ that functions to strengthen and organize the ECM to endure longitudinal tensile forces. Fibrillar collagen is a noncentromeric molecule, therefore making it ideal for a specific luminescence

\footnotetext{
${ }^{1}$ Department of Women's Health, Research Institute for Women's Health, Eberhard Karls University Tübingen, Tübingen, Germany.

${ }^{2}$ Department of Cell and Tissue Engineering, Fraunhofer Institute for Interfacial Engineering and Biotechnology (IGB), Stuttgart, Germany.

${ }^{3}$ Department of Medicine/Cardiology, Cardiovascular Research Laboratories, University of California, Los Angeles, California.

(C) Michael G. Monaghan, et al., 2016; Published by Mary Ann Liebert, Inc. This Open Access article is distributed under the terms of the Creative Commons Attribution Noncommercial License (http://creativecommons.org/licenses/by-nc/4.0/) which permits any noncommercial use, distribution, and reproduction in any medium, provided the original author(s) and the source are credited.
} 
phenomenon of multiphoton imaging: second harmonic generation $(\mathrm{SHG}){ }^{13,14}$ This occurs in highly polarizable materials with nonsymmetric molecular organization ${ }^{15}$ and was first demonstrated in crystalline quartz by Kleinman in 1962. ${ }^{16}$ SHG excitation depends on the simultaneous interaction of two photons with target molecules. The two photons are not absorbed; rather, the energy and coherence of the laser light are preserved to generate one photon of twice the energy. The wavelength of the emitted light is half that of the NIR excitation source, or in other words, the frequency of the incident NIR is doubled by passing through the noncentrosymmetric structures. ${ }^{15}$

Freshly obtained tissue samples are suitable for multiphoton and SHG imaging, which enables tissue evaluation under unaltered physiological conditions and allows immediate access to structural information. The possibility to perform microscopy on fresh unprocessed tissues is of great interest clinically to identify tissue structures without timeconsuming preservation methods. ${ }^{8,17}$ Nonlinear microscopy can be also performed on cryopreserved tissues, but the formation of ice crystals during the preservation process creates morphological distortions, which is a limiting factor. ${ }^{18,19}$ Preservation of tissues via fixation and paraffin embedding maintains an adequate tissue morphology comparable to fresh tissue and enables prolonged storage of the tissue for years. ${ }^{20}$ Paraffin embedding also allows a detailed investigation of tissues at various depths via serial sectioning. However, paraffin wax and fixation agents imbibe autofluorescence within tissues, which results in a reduced nonlinear image quality. ${ }^{21,22}$ Based on this, multiphoton imaging is more often done with fresh biopsies before paraffin embedding and hematoxylin and eosin $(\mathrm{H} \& \mathrm{E})$ staining to receive an additional preprocessing result. $^{23,24}$ It would be extremely advantageous to perform satisfactory multiphoton imaging of tissue samples archived in pathology laboratories for possible reinvestigations of tissues upon which minimum tissue volume is available, especially in rare diseases so as to increase the number of samples to gain statistically noteworthy data. Therefore, the aim of this study was to enable adequate multiphoton imaging of paraffinembedded tissue sections by applying clearing protocols to reduce the influence of the paraffin on the emission profiles of sections. We also sought to investigate if multiphoton imaging can be performed on histologically stained sections.

\section{Materials and Methods}

Unless otherwise stated, all materials and reagents were obtained from Sigma-Aldrich (Taufkirchen, Germany).

\section{Multiphoton-induced laser scanning microscopy}

In this study, we used a custom-made multiphoton laser system (Fig. 1; JenLab GmbH, Jena, Germany). A tunable titanium-sapphire laser with a pulse repetition rate of $80 \mathrm{MHz}$ and tunable wavelength excitation range of 720 920 nm (MaiTai XF 1; Spectra Physics, Santa Clara, CA) was used for multiphoton imaging. Multiphoton and SHG imaging was performed (40× oil immersion objective: N.A 1.3; Carl Zeiss, Jena, Germany) at an acquisition time of $48.5 \mathrm{~s}$ and a pixel resolution of $512 \times 512$. The system was equipped with two photomultiplier tubes (PMTs), 425-509 and $\leq 425 \mathrm{~nm}$, therefore enabling multiphoton and SHG imaging simultaneously with this optical setup. The atten- uator of the laser was set to $20 \mathrm{~mW}$ and the PMTs to $1000 \mathrm{~V}$ for contrast and $52.7 \%$ for brightness. These settings were maintained for all multiphoton and SHG imaging in this study.

\section{Tissue processing and routine histology}

As a model tissue for imaging, porcine pulmonary semilunar valves were excised from hearts obtained from a local slaughterhouse, rinsed twice in PBS, and cut into three leaflets before further processing. These fresh leaflets were positioned on coverslips for multiphoton imaging. After imaging, the tissues were fixed in $4 \%$ PFA at $4^{\circ} \mathrm{C}$ overnight and subsequently embedded in paraffin. Paraffin-embedded tissues were later sectioned into $3-\mu \mathrm{m}$-thick sections and deparaffinized in xylene, rehydrated in gradients of ethanol as previously described, ${ }^{25}$ mounted using an aqueous mounting medium (Dako Faramount; Dako Deutschland $\mathrm{GmbH}$, Hamburg, Germany), and sealed with a coverslip. As a control, untreated paraffin sections (without deparaffinization and without rehydration) were also mounted with coverslips using Dako Faramount. All samples were left at room temperature overnight before imaging. Imaging was performed at the ventricularis, spongiosa, and fibrosa of the heart valve leaflets.

Routine H\&E staining was performed on the deparaffinized tissues, followed by dehydration via increasing gradients of alcohol and mounting the sections with a coverslip using Isomount 2000 (VWR GmbH, Darmstadt, Germany). Slides were left overnight before multiphoton imaging. Bright-field images of the H\&E-stained sections were taken with the Observer.Z1 (Carl Zeiss, Oberkochen, Germany) using a $40 \times$ water immersion objective (N.A 1.1; Carl Zeiss, Jena, Germany).

\section{Destaining of H\&E-stained sections}

H\&E-stained and mounted slides were immersed in $100 \%$ xylene on an orbital shaker overnight at room temperature to detach the coverslips. Following this, the slides were washed in fresh $100 \%$ xylene for $10 \mathrm{~min}$ to remove any excess mounting media. The slides were then rehydrated in gradients of alcohol. Hematoxylin was removed by incubation in $0.25 \%$ hydrochloric acid in $50 \%$ ethanol for $\sim 3 \mathrm{~min}$, followed by washing in distilled water for $3 \mathrm{~min}$. Eosin was removed using a mixture of $1.5 \%$ ammonium hydroxide in $70 \%$ ethanol for $\sim 3$ min. Afterward, samples were washed twice in distilled water for $5 \mathrm{~min}$ and mounted with an aqueous mounting medium (Dako Faramount) for both multiphoton imaging and routine bright-field microscopy.

\section{Calculation of acquired image qualities}

Gray value $(\mathrm{GV})$ intensities of the acquired images were determined and analyzed with ImageJ (freely available from www.nih.org). Briefly, the GV data from each image assign a value for the brightness of each pixel in the image and provide an overview of the distribution of the pixel brightness for the complete image. The mean of these $\mathrm{GVs}(\bar{x})$ is a metric of the intensity of an image [Eq. (1)]:

$$
G V I=\frac{1}{n} \sum_{i=1}^{n} x_{i}
$$



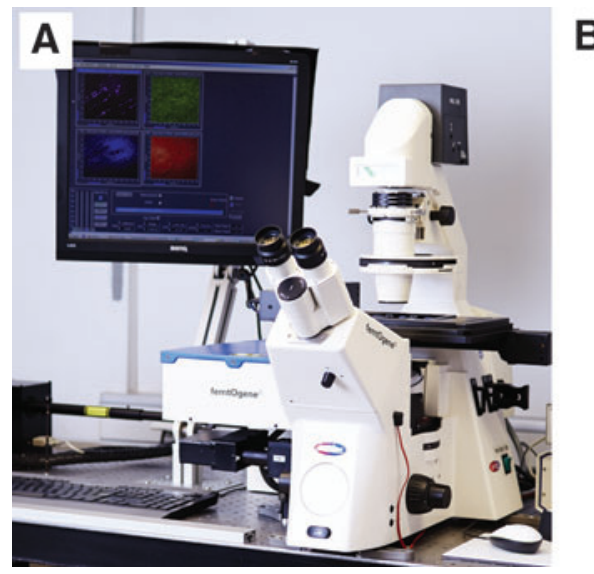

FIG. 1. Overview of the nonlinear femtosecond laser system used in this study. (A) Custom-built MP laser scanning MP/SHG imaging station. (B) Laser pathway setup with beam splitter to allow simultaneous acquisition of MP and SHG signals using PMTs with the detection of 425-509 and $\leq 425 \mathrm{~nm}$, respectively. MP, multiphoton; PMT, photomultiplier tube; SHG, second harmonic generation. Color images available online at www.liebertpub.com/tec
The standard deviation of these GVs $(s d v(x))$ represents the contrast of an image [Eq. (2)]:

$$
G V S D=\sqrt{\frac{1}{(n-1)} \cdot \sum_{i=1}^{n}\left(x_{i}-\bar{x}\right)^{2}}
$$

In this instance, a high intensity represents a stronger emission intensity, and a high standard deviation within one image indicates a good separation between the true signal and background.

\section{Statistical analysis}

Normal data distribution was assessed using a ShapiroWilk test. For statistical analysis, the commercially available program GraphPad Prism 6 was used (GraphPad Software, Inc., La Jolla, CA). A one-way ANOVA or two-way ANOVA was performed when appropriate. $p<0.05$ was considered statistically significant.

\section{Results}

An increasing number of researchers are using multiphoton imaging as an analytical tool before routine histological processing. In the majority of the studies, multiphoton imaging is performed on sections before the beginning of a staining procedure, such as $\mathrm{H} \& \mathrm{E}$, to gather more information about a sample. ${ }^{26,27}$ In other cases, whole paraffin-embedded tissues have been clarified using various agents and imaged as one whole piece due to the advantageous penetration capabilities of multiphoton imaging. ${ }^{20}$ Here, we rehydrated
A
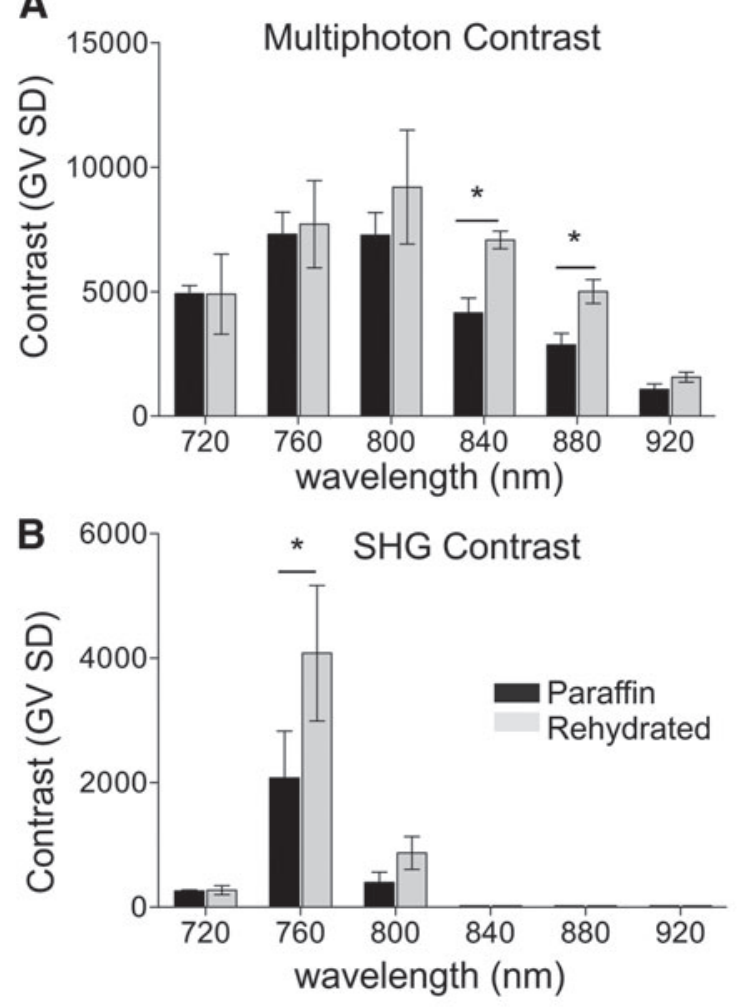
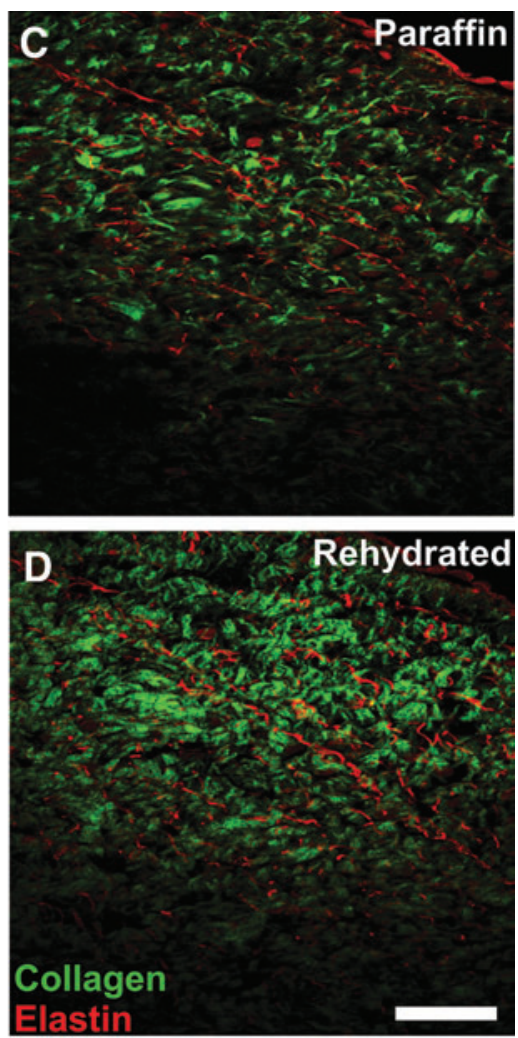

FIG. 2. (A) MP and (B) SHG signal contrast of porcine heart valves after excitation with 720-920 nm (GV SD = gray value standard deviation). Data are presented as mean \pm standard deviation, $n=4$. $*$ Statistically significant increase in the emission signal contrast of rehydrated sections compared with paraffin sections at the excitation wavelengths indicated, $p<0.05$. Merged MP and SHG images (C) before and (D) after rehydration. Scale bar equals $60 \mu \mathrm{m}$. Color images available online at www.liebertpub .com/tec 
paraffin-embedded tissue sections using a routine protocol to bring the samples to a physiological state for improved multiphoton and SHG imaging. Using the custom laser system in this study (Fig. 1), excitation wavelengths in the range of $760-840 \mathrm{~nm}$ provided the strongest contrast in the emission signals of the multiphoton channel, which can be mainly attributed to the elastic fibers in the heart valve sections (Fig. 2A). Bringing these sections to a rehydrated state gave significantly higher multiphoton emission signal contrasts (thereby reduced background and improved imaging) at excitation wavelengths of 840 and $880 \mathrm{~nm}$. However, the strongest multiphoton emission signal contrast was obtained at $800 \mathrm{~nm}$. Analysis of the effect of excitation wavelength on SHG signals is more profound, with the strongest contrast SHG emission signal obtained at an excitation of $760 \mathrm{~nm}$ (Fig. 2B) with both paraffin-embedded and rehydrated sections. The effect of rehydrating the paraffin sections also had a statistically significantly higher SHG emission signal value of contrast at this excitation compared with paraffinembedded sections. These results are reflected in the micrographs of the paraffin sections before and after rehydration (Fig. 2C, D). Notably, the signal of the SHG emission, reflective of the collagen content in the valve leaflet, has a much stronger contrast. Based on this result, simultaneous acquisition of the multiphoton and SHG emission signals could be obtained at an excitation wavelength of $760 \mathrm{~nm}$.

It was next sought to determine if it would be possible to acquire satisfactory multiphoton and SHG images of histologically stained sections. As H\&E is one of the most commonly used staining techniques in both research and pathology, paraffin sections were stained with $\mathrm{H} \& \mathrm{E}$ using standard protocols and then mounted for storage (Fig. 3A-C). Multiphoton and SHG images were acquired from these sections, which yielded extremely saturating emission signals, particularly in the multiphoton emission channel (Fig. 3D-F and see Supplementary Fig. S1 for monochromatic signals of
FIG. 3. (A-C) Bright-field images of H\&E-stained porcine leaflet sections, followed by (D-F) subsequent MP and SHG imaging of the same regions. Green represents collagen fibers, whereas red indicates elastic fibers. The same sections were destained and again subjected to (G-I) bright-field microscopy as well as (J-L) MP and SHG imaging. Scale bar equals $60 \mu \mathrm{m}$. H\&E, hematoxylin and eosin. Color images available online at www .liebertpub.com/tec
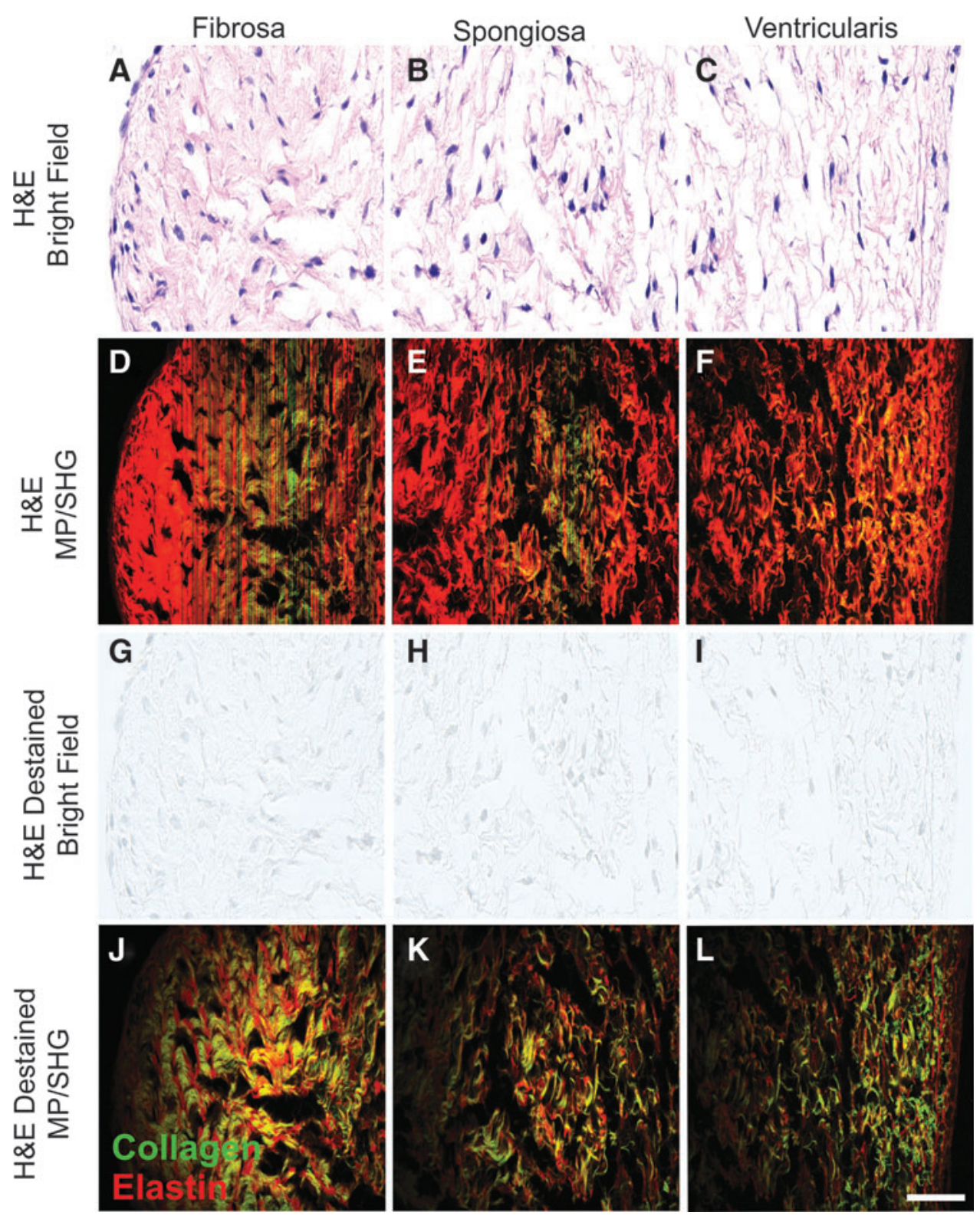

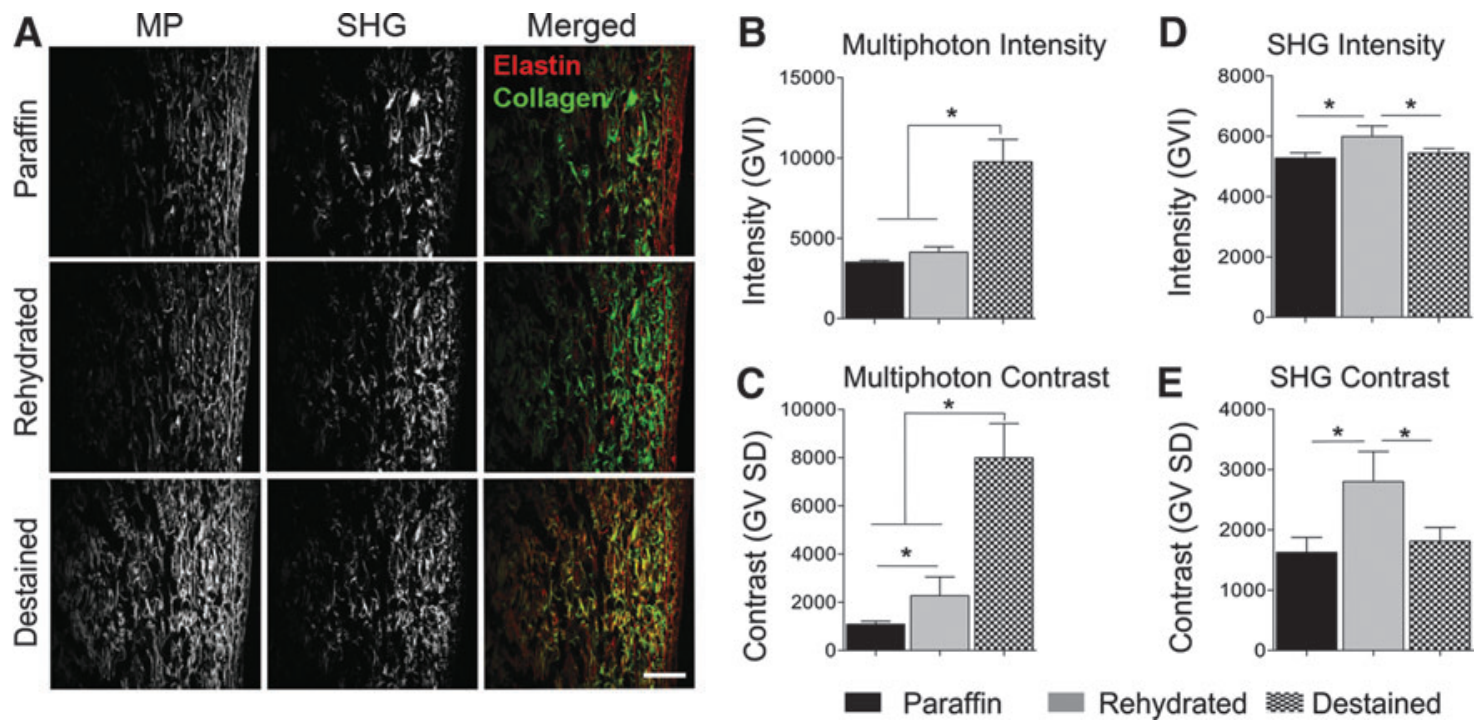

FIG. 4. (A) MP and SHG imaging of porcine leaflets while present in paraffin, deparaffinized rehydrated states, and destained H\&E sections. Merged images represent the MP (red) and SHG (green) channels. Scale bar equals $60 \mu \mathrm{m}$. Bar graphs represent $(\mathbf{B}, \mathbf{C}) \mathrm{MP}$ and $(\mathbf{D}, \mathbf{E}) \mathrm{SHG}$ intensities $(\mathrm{GVI}=$ gray value intensities $)$ and contrast $(\mathrm{GV}$ SD $=$ gray value standard deviation). Data are presented as mean \pm standard deviation, $n=8$. *Statistically significant difference between the groups indicated, $p<0.05$. Color images available online at www.liebertpub.com/tec

these images; Supplementary Data are available online at www.liebertpub.com/tec). These saturating signals in the multiphoton emission channel were also observed at lower laser excitation powers (Supplementary Fig. S2). Images obtained from these H\&E-stained sections were deemed unsuitable for interpretation of the ECM structure (namely collagen and elastic fibers). Using a destaining protocol based on acidic and alkaline dissolution of the histological dyes out of the tissue sections, we succeeded in destaining the tissue, whereby no dyes were visually present when imaged using bright-field microscopy (Fig. 3G-I). Satisfactory multiphoton and SHG imaging was attainable from these sections (Fig. 3J-L and see Supplementary Fig. S3 for monochromatic signals of these images) following the destaining protocol, whereby the tissue was brought again to a hydrated state as optimized for Figure 2. However, there was a statistically significant increase after the destaining protocol in the multiphoton signal intensity and contrast values compared to paraffin-embedded and rehydrated sections, most likely due to residual elements of the H\&E dyes (Fig. 4B, D). However, the SHG signal was significantly improved with a level closer to that of nonstained paraffin-embedded tissues. Rehydrated tissues remained to have the highest SHG detection with a significantly higher contrast and intensity compared with destained and paraffinembedded tissues (Fig. 4C, E).

\section{Discussion}

Noninvasive multiphoton and SHG imaging has already been used in the analysis of fresh biopsies, ${ }^{28}$ in vivo, ${ }^{1}$ and of cryopreserved tissues. ${ }^{18}$ However, it can sometimes be difficult to correctly orientate fresh tissues, and although multiphoton imaging extends the depth of imaging that is possible, it still has its limitations. ${ }^{29}$ Additionally, although cryosectioning can enable the storage of tissue without fixation, the rapid freezing processes can incur damage to the morphological structure of the tissues and ECM structures via water expansion, and therefore, a realistic representation of the tissue using nonlinear imaging is impaired. ${ }^{18}$ Paraffinembedded tissue, in contrast, maintains a relative representation of the morphological structure of the tissue. Therefore, many research groups have attempted clearing protocols to remove paraffin from tissue with the aim of maintaining the structural integrity of the tissue for multiphoton imaging. ${ }^{20}$

Here, we demonstrated that by simply removing paraffin during a standard staining protocol and bringing tissue sections to a rehydrated state, adequate multiphoton imaging can be performed on tissue sections. The effect of rehydration was most significant in the SHG emission channel, whereby the contrast was significantly increased when an excitation of $760 \mathrm{~nm}$ was applied. This could be for a couple of reasons. The physiological state of collagen is very important, and previously, the hydration state of collagen has been shown to have a significant effect on the mechanical properties of collagen fibers based on intermolecular forces between tropocollagen monomers. ${ }^{30}$ In addition, the environmental $\mathrm{pH}$ has a significant effect on the stability of the triple helix. ${ }^{31}$ Indeed, SHG has been demonstrated to radiate from the shell of collagen fibrils, rather than from its bulk, with physiological relevant changes in environmental ionic strength changing the intensity and nature of the SHG signal. $^{32}$ The same study found that paraformaldehyde-fixed tissues exhibited similar SHG signaling as fresh tissues at very low ionic concentrations $(0-2 \mathrm{mM} \mathrm{NaCl})$. Furthermore, SHG signaling decreased rapidly with increasing ionic concentrations, even at physiological levels. ${ }^{32}$ This is attributed to the swelling properties of collagen as immersing collagen in water can result in a $10-20 \%$ increase in the average lateral spacing between collagen triple helices. ${ }^{33}$ Although we do not have complete tissues of collagen, but rather histological sections, it can be taken that the hydration 
process causes the collagen in the leaflet tissue to swell and increase the SHG signal. Therefore, it is suggested that future imaging of fixed tissues and sections should be performed in water or solutions of extremely low ionic concentrations.

$\mathrm{H} \& \mathrm{E}$ staining is commonly used in animal histology and routine pathology. ${ }^{34}$ It is known that hematoxylin stains the nuclei in a purple/blue color, whereas eosin shows the extracellular and intracellular protein in pink. The significance of this study is that multiphoton and SHG imaging can be performed on sections that were previously stained histologically using a suitable destaining protocol. We identified that the quality of the images was drastically improved (although not ideal) after destaining, which allowed identification of ECM structures. Moreover, the destained sections did retain a rather high level of multiphoton fluorescence intensity and contrast. We did investigate the influence of mounting media on the multiphoton and SHG emission signals of deparaffinized sections but found no significant influence on the quality of the images obtained (Supplementary Fig. S4). Hematoxylin is nonfluorescent and generally stains the nuclei of cells; therefore, it can be taken that even if trace amounts of hematoxylin remain, they do not contribute to the increased multiphoton signal. Eosin, in contrast, accumulates in the cytoplasm and ECM and has been reported to contribute to emission signaling in fluorescence lifetime imaging microscopy (FLIM) and multiphoton microscopy. ${ }^{35,36}$ Observations in previous studies noted that from H\&E-stained cervical tissue, epithelial fluorescence originated from the extracellular and intracellular components, and not the nuclei, which emphasizes the contribution of eosin to the multiphoton fluorescence. ${ }^{36}$ It has also been reported that the spectral properties of eosin are varied upon binding with proteins, which could be used as an indirect way to distinguish between various grades of tissue based on fluorescent lifetimes. ${ }^{35}$ Our results here agree in that although the destaining protocol was successful in generating satisfactory micrographs, the multiphoton emission signals were significantly increased, most likely due to the presence of residual eosin in the tissue sections. However, SHG signaling was not significantly affected by the histological dyes.

\section{Conclusion}

Simultaneous multiphoton-induced autofluorescence and SHG imaging on paraffin-embedded sections was achieved in this study. We identified that rehydrating such sections can significantly improve the autofluorescence and SHG signal detection capabilities. H\&E staining significantly influences the multiphoton emission signals, even after a destaining protocol is applied. However, prior H\&E staining does not impact the SHG emission signaling after destaining even though eosin molecules are still present that affect the multiphoton emission signal. Our findings are important if researchers are to combine $\mathrm{H} \& \mathrm{E}$ staining with multiphoton imaging or reinvestigate fixed stained sections with nonlinear microscopy. This will enable the acquisition of additional data regarding the ECM on tissue sections that are being used for histological processing where limited tissue is available or only a small number of tissue sections have an area that is of interest to the researcher/pathologist and retrospective nonlinear imaging is desirable.

\section{Acknowledgments}

This work was supported by funding from the European Union's Seventh Framework Programme for research, technological development, and demonstration under Grant Agreement No. 331430 (to M.M.), as well as the Ministry of Science, Research and the Arts of Baden-Württemberg (33729.55-3/214 and SI-BW 01222-91), and the Deutsche Forschungsgemeinschaft (INST 2388/30-1, SCHE 701/7-1, SCHE 701/10-1) (all to K.S.-L.).

\section{Disclosure Statement}

No competing financial interests exist.

\section{References}

1. Ait El Madani, H., Tancrède-Bohin, E., Bensussan, A., Colonna, A., Dupuy, A., Bagot, M., and Pena, A.M. In vivo multiphoton imaging of human skin: assessment of topical corticosteroid-induced epidermis atrophy and depigmentation. J Biomed Opt 17, 026009, 2012.

2. Perry, S.W., Burke, R.M., and Brown, E.B. Two-photon and second harmonic microscopy in clinical and translational cancer research. Ann Biomed Eng 40, 277, 2012.

3. Vielreicher, M., Schürmann, S., Detsch, R., Schmidt, M.A., Buttgereit, A., Boccaccini, A., and Friedrich, O. Taking a deep look: modern microscopy technologies to optimize the design and functionality of biocompatible scaffolds for tissue engineering in regenerative medicine. J R Soc Interface 10, 20130263, 2013.

4. Basuki, J.S., Duong, H.T., Macmillan, A., Erlich, R.B., Esser, L., Akerfeldt, M.C., Whan, R.M., Kavallaris, M., Boyer, C., and Davis, T.P. Using fluorescence lifetime imaging microscopy to monitor theranostic nanoparticle uptake and intracellular doxorubicin release. ACS Nano 7, 10175, 2013.

5. Fritze, O., Schleicher, M., König, K., Schenke-Layland, K., Stock, U., and Harasztosi, C. Facilitated noninvasive visualization of collagen and elastin in blood vessels. Tissue Eng Part C Methods 16, 705, 2010.

6. Göppert-Mayer, M. Über elementarakte mit zwei quantensprüngen. Ann Phy 401, 273, 1931.

7. Kaiser, W., and Garrett, C.G.B. Two-photon excitation in $\mathrm{CaF}_{2}: \mathrm{Eu}^{2+}$. Phys Rev Lett 7, 229, 1961.

8. Benninger, R.K.P., and Piston, D.W. Two-photon excitation microscopy for the study of living cells and tissues. Curr Protoc Stem Cell Biol Chapter 4, 1, 2013.

9. Wang, B.G., König, K., and Halbhuber, K.J. Two-photon microscopy of deep intravital tissues and its merits in clinical research. J Microsc 238, 1, 2010.

10. Hinderer, S., Shena, N., Ringuette, L.J., Hansmann, J., Reinhardt, D.P., Brucker, S.Y., Davis, E.C., and SchenkeLayland, K. In vitro elastogenesis: instructing human vascular smooth muscle cells to generate an elastic fiber-containing extracellular matrix scaffold. Biomed Mater 10, 034102, 2015.

11. Roberts, T.J. Contribution of elastic tissues to the mechanics and energetics of muscle function during movement. J Exp Biol 219, 266, 2016.

12. Alberts, B., Johnson, A., and Lewis, J. Molecular Biology of the Cell, 4th ed. New York: Garland Science, 2002.

13. Ranjit, S., Dvornikov, A., Stakic, M., Hong, S.H., Levi, M., Evans, R.M., and Gratton, E. Imaging fibrosis and separating collagens using second harmonic generation and phasor approach to fluorescence lifetime imaging. Sci Rep 5, 13378, 2015. 
14. Tsai, M.R., Chiu, Y.W., Lo, M.T., and Sun, C.K. Secondharmonic generation imaging of collagen fibers in myocardium for atrial fibrillation diagnosis. J Biomed Opt 15, 026002, 2010.

15. Campagnola, P.J., and Loew, L.M. Second-harmonic imaging microscopy for visualizing biomolecular arrays in cells, tissues and organisms. Nat Biotechnol 21, 1356, 2003.

16. Kleinman, D.A. Theory of second harmonic generation of light. Phys Rev 128, 1761, 1962.

17. Torres, R., Vesuna, S., and Levene, M.J. High-resolution, 2- and 3-dimensional imaging of uncut, unembedded tissue biopsy samples. Arch Pathol Lab Med 138, 395, 2013.

18. Schenke-Layland, K., Madershahian, N., Riemann, I., Starcher, B., Halbhuber, K.J., König, K., and Stock, U.A. Impact of cryopreservation on extracellular matrix structures of heart valve leaflets. Ann Thorac Surg 81, 918, 2006.

19. Theodossiou, T.A., Thrasivoulou, C., Ekwobi, C., and Becker, D.L. Second harmonic generation confocal microscopy of collagen type I from rat tendon cryosections. Biophys J 91, 4665, 2006.

20. Wilson, J.W., Degan, S., Warren, W.S., and Fischer, M.C. Optical clearing of archive-compatible paraffin embedded tissue for multiphoton microscopy. Biomed Opt Express 3, 2752, 2012.

21. Leischner, U., Schierloh, T., Zieglgänsberger, W., and Dodt, H.U. Formalin-induced fluorescence reveals cell shape and morphology in biological tissue samples. PLoS One 5, e10391, 2010.

22. Baschong, W., Suetterlin, R., and Laeng, R.H. Control of autofluorescence of archival formaldehyde-fixed, paraffinembedded tissue in confocal laser scanning microscopy. J Histochem Cytochem 49, 1565, 2001.

23. Jain, M., Robinson, B.D., Scherr, D.S., Sterling, J., Lee, M.M., Wysock, J., Rubin, M.A., Maxfield, F.R., Zipfel, W.R., Webb, W.W., and Mukherjee, S. Multiphoton microscopy in the evaluation of human bladder biopsies. Arch Pathol Lab Med 136, 517, 2012.

24. Wong, S., Nathanson, M.H., Chen, J., and Jain, D. Evaluation of barrett esophagus by multiphoton microscopy. Arch Pathol Lab Med 138, 204, 2014.

25. Monaghan, M., Browne, S., Schenke-Layland, K., and Pandit, A. A collagen-based scaffold delivering exogenous microrna-29B to modulate extracellular matrix remodeling. Mol Ther 22, 786, 2014.

26. Chen, A.C.H., McNeilly, C., Liu, A.P.Y., Flaim, C.J., Cuttle, L., Kendall, M., Kimble, R.M., Shimizu, H., and McMillan, J.R. Second harmonic generation and multiphoton microscopic detection of collagen without the need for species specific antibodies. Burns 37, 1001, 2011.
27. Kottmann, R.M., Sharp, J., Owens, K., Salzman, P., Xiao, G.Q., Phipps, R.P., Sime, P.J., Brown, E.B., and Perry, S.W. Second harmonic generation microscopy reveals altered collagen microstructure in usual interstitial pneumonia versus healthy lung. Respir Res 16, 61, 2015.

28. König, K., Breunig, H.G., Bückle, R., Kellner-Höfer, M., Weinigel, M., Büttner, E., Sterry, W., and Lademann, J. Optical skin biopsies by clinical CARS and multiphoton fluorescence/SHG tomography. Laser Phys Lett 8, 465, 2011.

29. Theer, P., and Denk, W. On the fundamental imaging-depth limit in two-photon microscopy. J Opt Soc Am A Opt Image Sci Vis 23, 3139, 2006.

30. Grant, C.A., Brockwell, D.J., Radford, S.E., and Thomson, N.H. Tuning the elastic modulus of hydrated collagen fibrils. Biophys J 97, 2985, 2009.

31. Leikina, E., Mertts, M.V., Kuznetsova, N., and Leikin, S. Type I collagen is thermally unstable at body temperature. Proc Natl Acad Sci U S A 99, 1314, 2002.

32. Williams, R.M., Zipfel, W.R., and Webb, W.W. Interpreting second-harmonic generation images of collagen I fibrils. Biophys J 88, 1377, 2005.

33. Price, R.I., Lees, S., and Kirschner, D.A. X-ray diffraction analysis of tendon collagen at ambient and cryogenic temperatures: role of hydration. Int J Biol Macromol 20, 23, 1997.

34. Young, B., O'Dowd, G., and Woodford, P. Wheater's Functional Histology: A Text and Colour Atlas, 6th ed. London: Churchill Livingstone, 2013.

35. Birla, L., Cristian, A.-M., and Hillebrand, M. Absorption and steady state fluorescence study of interaction between eosin and bovine serum albumin. Spectrochim Acta Mol Biomol Spectrosc 60, 551, 2004.

36. Gu, J., Fu, C.Y., Ng, B.K., Gulam Razul, S., and Lim, S.K. Quantitative diagnosis of cervical neoplasia using fluorescence lifetime imaging on haematoxylin and eosin stained tissue sections. J Biophotonics 7, 483, 2014.

Address correspondence to: Katja Schenke-Layland, PhD, MSc

Department of Women's Health

Research Institute for Women's Health Eberhard Karls University Tübingen

Silcherstr. $7 / 1$

Tübingen 72076

Germany

E-mail: katja.schenke-layland@med.uni-tuebingen.de

Received: February 24, 2016

Accepted: March 24, 2016

Online Publication Date: April 22, 2016 